\title{
Visibility Assessment of Latent Fingerprints on Challenging Substrates in Spectroscopic Scans
}

\author{
Mario Hildebrandt, Andrey Makrushin, Kun Qian, and Jana Dittmann \\ Research Group on Multimedia and Security, Otto-von-Guericke University of Magdeburg, \\ Universitaetsplatz 2, 39106 Magdeburg, Germany \\ \{hildebrandt, makrushin, kqian, dittmann\} @iti.cs.uni-magdeburg.de
}

\begin{abstract}
Our objectives for crime scene forensics are to find the substrates on which finger traces are visible in limited ranges of the electromagnetic spectrum using UV-VIS reflection spectroscopy and to determine the optimal ranges in the interval from 163 to $844 \mathrm{~nm}$. We subjectively assess the visibility of fingerprints within detailed scans with a resolution of 500 ppi and compare the results with those of an automatic visibility assessment based on the streakiness score. Ten different substrates are evaluated, each with three fingerprints from different donors. Streakiness score is confirmed to be a suitable fingerprint visibility indicator on non-structured substrates. We identify two substrates, namely metallic paint and blued metal, on which ridge lines become visible exclusively in UV range from 200 to $400 \mathrm{~nm}$ and from 210 to $300 \mathrm{~nm}$ correspondingly.
\end{abstract}

Keywords: Forensics, latent fingerprints, UV-VIS spectroscopy, fingerprint visibility assessment.

\section{Extended Abstract}

As the most common form of trace evidence left at crime scenes, latent fingerprints pose the most challenges for forensic experts [1]. Since they are present rather often yet invisible unless brought out with chemical and/or physical processes designed to enhance the visibility of fingerprint residues [2] (e.g. fuming, powdering, or deposition of chemical reagent), it is important to identify substrate dependent technique or reagent and the appropriate processing sequence for successful enhancement.

In today's crime scene forensics three kinds of substrate properties are known [2][3]: 1) porous substrates (e.g. paper) are absorbent and provide good adhesion for latent fingerprints; 2) non-porous substrates (e.g. glass) do not absorb, so latent fingerprints on such substrates are more susceptible to damage; 3) semi-porous substrates (e.g. glossy cardboard) both resist and absorb fingerprint residue, so it may or may not soak in depending on its viscosity as well as on the absorbent properties of the substrates. Since substrates have different characteristics regarding the absorption of fingerprint residues and light reflection, different fingerprint development techniques are required. On challenging substrates these techniques have an invasive nature as they involve applying alien substances on the fingerprint residue, thus causing 
the potential risk of compromising the evidence. Hence, various non-invasive approaches are developed to visualize latent fingerprints, e.g. by using various sensors and light sources [4], or Chromatic White Light (CWL) sensors [3][5][6]. It is common that substrates reflect specular light differently depending on the wavelength of the light source. This effect can be used to render fingerprint residues visible and to acquire them in detailed scans. However, inherent properties of substrates (e.g. translucency or structure) might interfere with the scanning, making the identification of the substrate of crucial importance.

We apply the spectroscopic acquisition with a lateral resolution of 500 ppi in the range from 163 to $844 \mathrm{~nm}$ on latent fingerprints deposited on ten substrates and then assess the fingerprint visibility in those detailed scans subjectively and by calculating the streakiness scores [5].

In the subjective visibility evaluation of the fingerprint a label corresponding to its suitability for forensic investigations is assigned. In particular, we distinguish between four classes of fingerprint visibility: 1) invisible, i.e. fingerprint is not perceptible for the naked eye; 2) weak pattern, i.e. fingerprint is partially visible but not usable for forensic investigations; 3 ) fair pattern, i.e. fingerprint is visible but covered by the substrate's texture or structure, and this usually requires longer investigation time; 4) perfect pattern, i.e. fingerprint can be easily segmented using binarization.

The streakiness score $S$ [5] is used as the objective visibility measure. However, in contrast to [5] we do not use substrate specific filters therefore achieve fully automatic processing. The basic idea is assigning $S$ to determine the visibility of the fingerprint at each particular wavelength. Since $S$ is the relative number of pixels in ridge lines in a binarized fingerprint pattern, its value rests in the interval $[0,1]$, where 0 denotes a completely invisible fingerprint pattern and 1 a perfectly visible one. It relies on the assumption that a fingerprint forms a striated pattern consisting of local ridge-valley patterns with constant frequencies and directions. A preprocessing in the Fourier domain is applied to enhance the ridge-valley pattern, afterwards an enhancement based on the approach from [7] is applied. The first step consists of multiplying the Fourier representation with a ring filter around its origin (see [8]) followed by a zero-mean normalization. The second step consists of estimating local orientations and a corresponding reliability map, both based on gradients. Furthermore, local frequencies are determined for reliable orientations. This is the foundation for locally applying corresponding Gabor filters for the enhancement of the ridge pattern. At last, the filtered image is binarized to calculate the streakiness score.

As a benchmark, we adapt the concept of differential images from [6] to eliminate possible interference introduced by the substrate and compare the visibility of fingerprints on original and differential images. On cooperative substrates the fingerprint visibility on original and differential images is expected to be equal. On challenging substrates with a constant reflection the originally invisible fingerprints become visible in differential scans. On challenging substrates with a random reflection the visibility is not expected to increase. The original approach subtracts the data from the same area of the substrate without a fingerprint. In our proposed approach, the image with the fingerprint is divided by the values from the images without the fingerprint: The intensity image $I\left(\lambda_{w}\right)$ at one particular wavelength $\lambda_{w}$ in the interval $[163,844] \mathrm{nm}$ 
with $w \in[0,2047]$ can be determined by $I\left(\lambda_{w}\right)=R\left(\lambda_{w}\right) \cdot S\left(\lambda_{w}\right) \cdot \operatorname{Sen} s\left(\lambda_{w}\right)$, where $R\left(\lambda_{w}\right)$ is the reflection of the measured material, $S\left(\lambda_{w}\right)$ is the spectral distribution of the light source, and $\operatorname{Sen} s\left(\lambda_{w}\right)$ is the sensitivity of the spectrometer. Then $R\left(\lambda_{w}\right)$ can be divided into the reflection of the substrate $R_{s}\left(\lambda_{w}\right)$ and the specific absorption and fluorescence of the fingerprint residue $R_{f}\left(\lambda_{w}\right)$. By performing a division of $I\left(\lambda_{w}\right)$ by the spectral data from a reference scan $I_{r e f}\left(\lambda_{w}\right), S\left(\lambda_{w}\right)$ and $\operatorname{Sen} s\left(\lambda_{w}\right)$, as well as $R_{s}\left(\lambda_{w}\right)$ are eliminated, leading to an enhanced contrast.

In total we evaluate ten substrates that are common at crime scenes as summarized in Table 1, each with three fingerprints from different donors. The substrates are divided into the classes cooperative, moderate and challenging based on observations for the CWL sensor [6]. On cooperative non-structured and non-textured substrates we can assume the following ranges streakiness scores $S$ for the subjective visibility classes: $S<0.1$ indicates an invisible fingerprint, $0.1<S<0.3$ usually corresponds with a weak pattern, $0.3<S<0.5$ indicates a fair pattern which probably contains smeared areas, $S>0.5$ indicates a good to perfect fingerprint visibility. However, since we do not apply substrate specific filters those ranges do not apply on moderate and challenging substrates because higher values of streakiness scores are likely to be caused by surface properties.

Table 1. Substrates, integration times and subjective evaluation results of original and differential images (*** - perfect, ** - good, * - weak, and o - invisible fingerprint pattern)

\begin{tabular}{|c|c|c|c|c|c|c|c|}
\hline \multirow[t]{2}{*}{ Substrate } & \multirow{2}{*}{$\begin{array}{l}\text { Integrati } \\
\text { on } \\
\text { time } \\
(\mathrm{ms})\end{array}$} & \multicolumn{2}{|c|}{ Original image } & \multicolumn{2}{|c|}{ Differential image } & \multirow{2}{*}{$\begin{array}{l}\text { Compariso } \\
\mathrm{n} \text { to } \\
\text { CWL [9] }\end{array}$} & \multirow[b]{2}{*}{$\begin{array}{l}\text { Substrate } \\
\text { property }\end{array}$} \\
\hline & & $\begin{array}{l}\text { Wavelength } \\
\text { range }(\mathrm{nm})\end{array}$ & Quality & $\begin{array}{l}\text { Wavelength } \\
\text { range }(\mathrm{nm})\end{array}$ & Quality & & \\
\hline $\begin{array}{l}\text { White } \\
\text { furniture } \\
\text { surface }\end{array}$ & 100 & $163-844$ & $* * *$ & $163-844$ & $* * *$ & identical & $\begin{array}{l}\text { non-porous, } \\
\text { cooperative }\end{array}$ \\
\hline $\begin{array}{l}\text { Metallic } \\
\text { paint }\end{array}$ & 100 & $200-385$ & $* * *$ & 195-385 & $* * *$ & better & $\begin{array}{l}\text { non-porous, } \\
\text { challenging }\end{array}$ \\
\hline Glass & 50 & $210-710$ & $* * *$ & $200-844$ & $* * *$ & identical & $\begin{array}{l}\text { non-porous } \\
\text { cooperative }\end{array}$ \\
\hline $\begin{array}{l}\text { Copy } \\
\text { paper }\end{array}$ & 250 & $220-250$ & $*$ & $220-250$ & $* *$ & $\begin{array}{l}\text { identical/b } \\
\text { etter }\end{array}$ & $\begin{array}{l}\text { porous, } \\
\text { challenging }\end{array}$ \\
\hline $\begin{array}{l}5 \text { Euro } \\
\text { cent coin }\end{array}$ & 100 & $220-520$ & $* * *$ & $220-844$ & $* * *$ & $\begin{array}{l}\text { identical } \\
\text { /better }\end{array}$ & $\begin{array}{l}\text { non-porous } \\
\text { cooperative }\end{array}$ \\
\hline $\begin{array}{l}\text { Blued } \\
\text { metal }\end{array}$ & 500 & $210-300$ & $*$ & $210-300$ & $* *$ & better & $\begin{array}{l}\begin{array}{l}\text { semi-porous, } \\
\text { challenging }\end{array} \\
\end{array}$ \\
\hline $\begin{array}{l}\text { Non- } \\
\text { metallic } \\
\text { paint }\end{array}$ & 250 & 163-844 & $\mathrm{O}$ & $200-220$ & $* / * *$ & $\begin{array}{l}\text { identical } \\
\text { /worse }\end{array}$ & $\begin{array}{l}\text { non-porous, } \\
\text { challenging }\end{array}$ \\
\hline $\begin{array}{l}\text { Brushed } \\
\text { stainless } \\
\text { steel }\end{array}$ & 100 & $163-844$ & $* / * *$ & $210-270$ & $* *$ & worse & $\begin{array}{l}\text { non-porous, } \\
\text { moderate }\end{array}$ \\
\hline $\begin{array}{l}\text { Beech } \\
\text { veneer }\end{array}$ & 100 & 163-844 & $* *$ & $163-844$ & $* * *$ & identical & $\begin{array}{l}\text { non-porous, } \\
\text { moderate }\end{array}$ \\
\hline $\begin{array}{l}\text { Golden } \\
\text { oak } \\
\text { veneer }\end{array}$ & 250 & $163-844$ & $\mathrm{o} / *$ & $163-844$ & $\mathrm{o} / *$ & worse & $\begin{array}{l}\text { non-porous, } \\
\text { moderate }\end{array}$ \\
\hline
\end{tabular}


On cooperative substrates (white furniture surface, glass and 5 Euro cent coin) the UV-VIS spectroscopy images yield satisfactory visibility throughout the entire spectrum with $S$ exceeding 0.5 . On copy paper the images are equally unusable as those captured using a CWL sensor. On beech veneer the visibility is identical with that on CWL images. On non-metallic paint, brushed stainless steel and golden oak veneer the visibility is slightly worse than that of a CWL sensor. The calculation of $S$ on structured surfaces (beech veneer, golden oak veneer, and brushed stainless steel) makes no sense since the streakiness of a surface dominates that of a fingerprint. Most notably, the spectrometer acquires a perfect fingerprint image within the UV band on metallic paint, whereas the transparent layer causes non-deterministic noise while using a CWL sensor [6]. Here, $S$ yields 0.7 between 200 and $400 \mathrm{~nm}$ and 0.05 for the spectrum of visible light. This can be explained by the fact that the covering transparent paint is non-transparent for UV radiation. On blued metal, fresh fingerprints are slightly visible in the UV band, which is an improvement compared to a CWL sensor.

The important conclusion is that the streakiness score generally reflects judgments of human experts about the fingerprint visibility. However, in order to extract meaningful streakiness scores a substrate specific preprocessing is necessary.

Acknowledgement. The authors wish to thank Michael Ulrich for the list of common substrates from crime scenes. The work in this paper has been funded in part by the German Federal Ministry of Education and Science (BMBF) through the Research Program under Contract No. FKZ: 13N10818.

\section{References}

1. Champod, C., et al.: Fingerprints and other ridge skin impressions. CRC Press (2004)

2. Scientific Working Group on Friction Ridge Analysis, Study and Technology (SWGFAST) et al.: The fingerprint sourcebook, U.S. Department of Justice (2011)

3. Makrushin, A., et al.: Advanced techniques for latent fingerprint detection and validation using a CWL device. In: Proc. SPIE, vol. 8436, p. 84360Y (2012)

4. Bleay, S.M., Sears, V.G., et al.: Fingerprint Source Book, Home Office (2012), https: / /www.gov.uk/government/publications / fingerprint-source-book

5. Makrushin, A., et al.: Visibility enhancement and validation of segmented latent fingerprints in crime scene forensics. In: Proc. SPIE, vol. 8665, p. 866508 (2013)

6. Hildebrandt, M., et al.: Benchmarking contact-less surface measurement devices for fingerprint acquisition in forensic investigations: results for a differential scan approach with a chromatic white light sensor. In: Proc. DSP 2011, pp. 1-6 (2011)

7. Hong, L., Wan, Y., Jain, A.: Fingerprint Image Enhancement: Algorithm and Performance Evaluation. IEEE Trans. on PAMI 30, 777-789 (1998)

8. Wu, C., Tulyakov, S., Govindaraju, V.: Image quality measures for fingerprint image enhancement. In: Gunsel, B., Jain, A.K., Tekalp, A.M., Sankur, B. (eds.) MRCS 2006. LNCS, vol. 4105, pp. 215-222. Springer, Heidelberg (2006) 